Supplementary Information

\title{
Improving Lithium Metal Composite Anodes with Seeding and Pillaring Effects of Silicon Nanoparticles
}

Hansen Wang, Xia Cao, Hanke Gu, Yayuan Liu, Yanbin Li, Zewen Zhang, William Huang, Hongxia Wang, Jiangyan Wang, Wu Xu, Ji-Guang Zhang, \& Yi Cui*

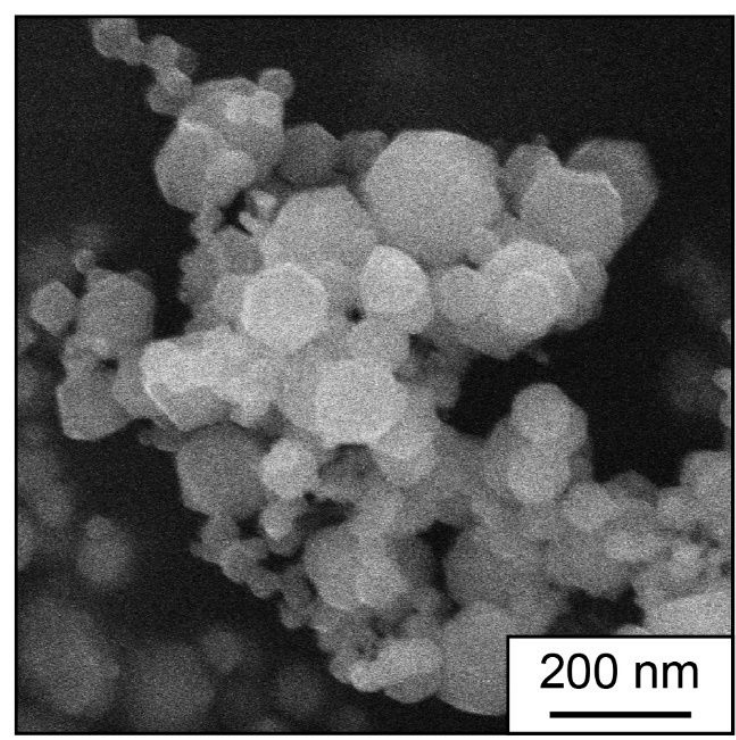

Figure S1. SEM image of the Si nanoparticles with diameters around $100 \mathrm{~nm}$.
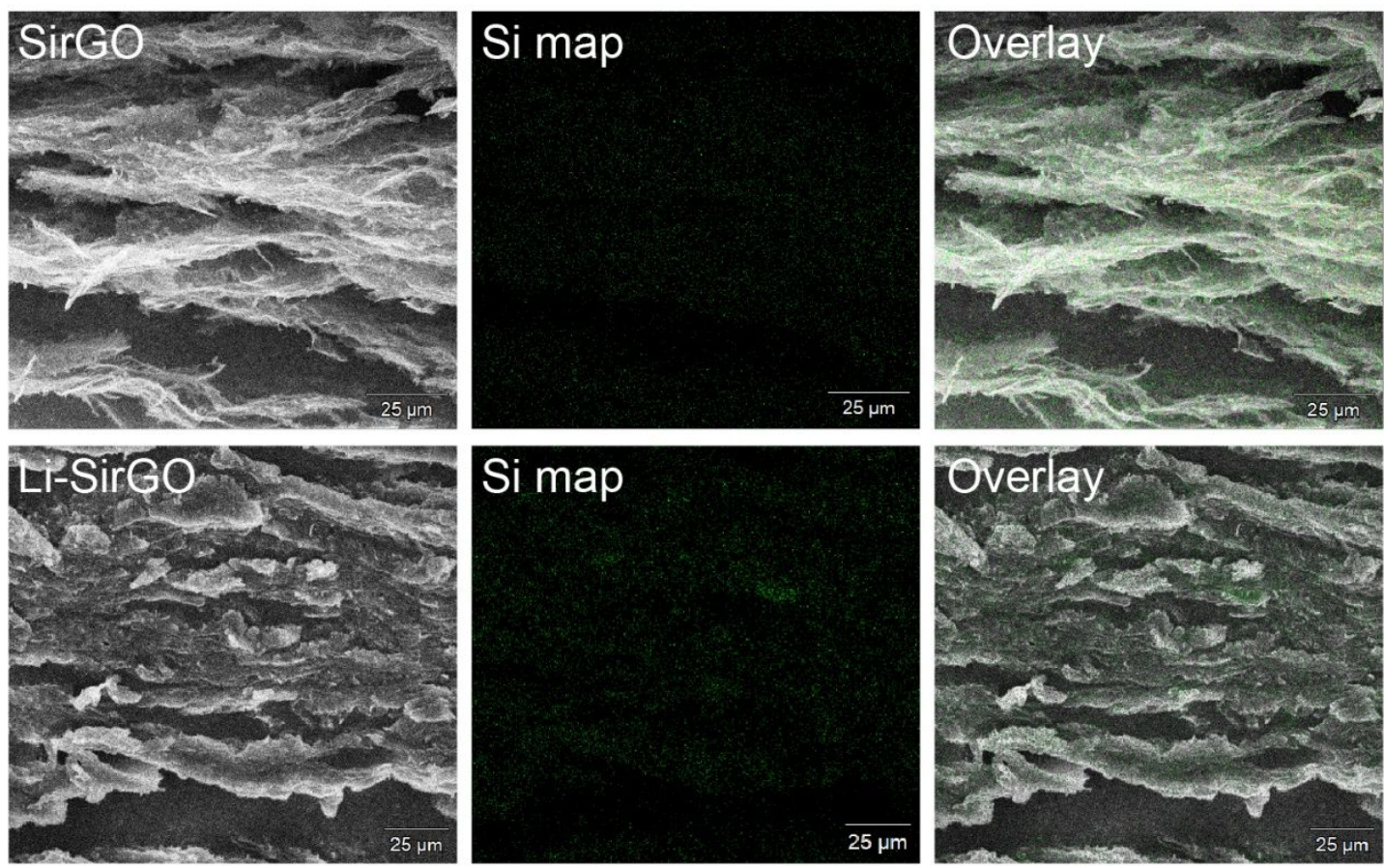

Figure S2. SEM images and SEM-EDS mapping of Si on the cross-section of SirGO and LiSirGO films. 

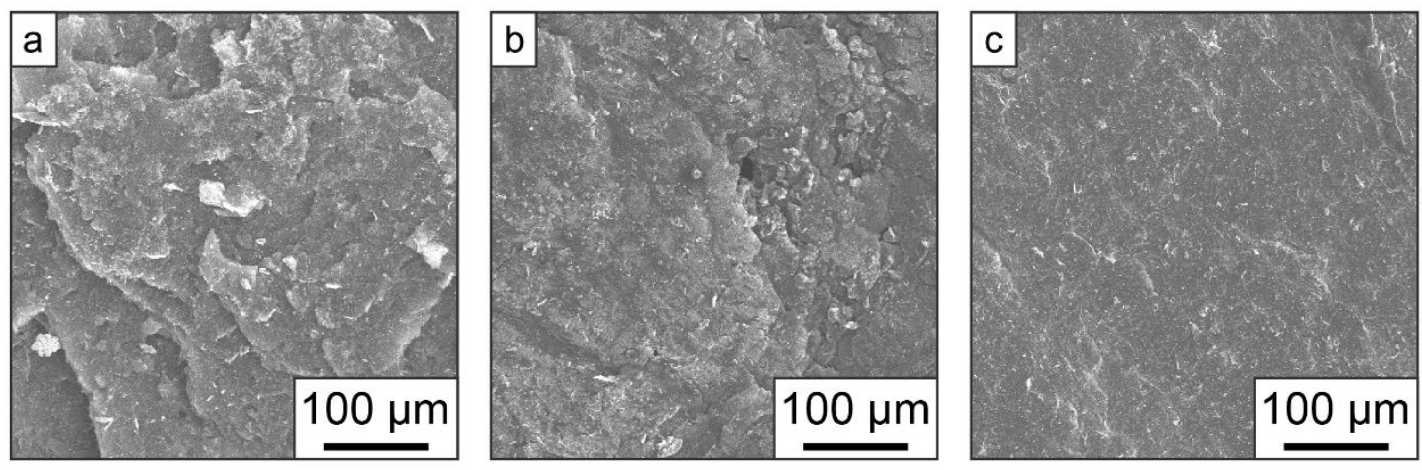

Figure S3. SEM images of the top-views of SiGO (a), SirGO (b) and Li-SirGO (c) films.

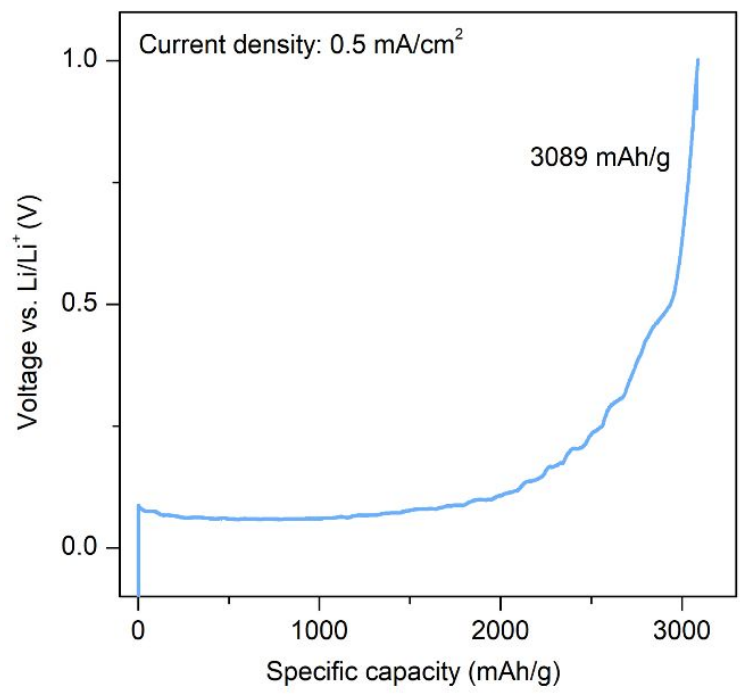

Figure S4. Specific capacity of Li-SirGO composite anode.

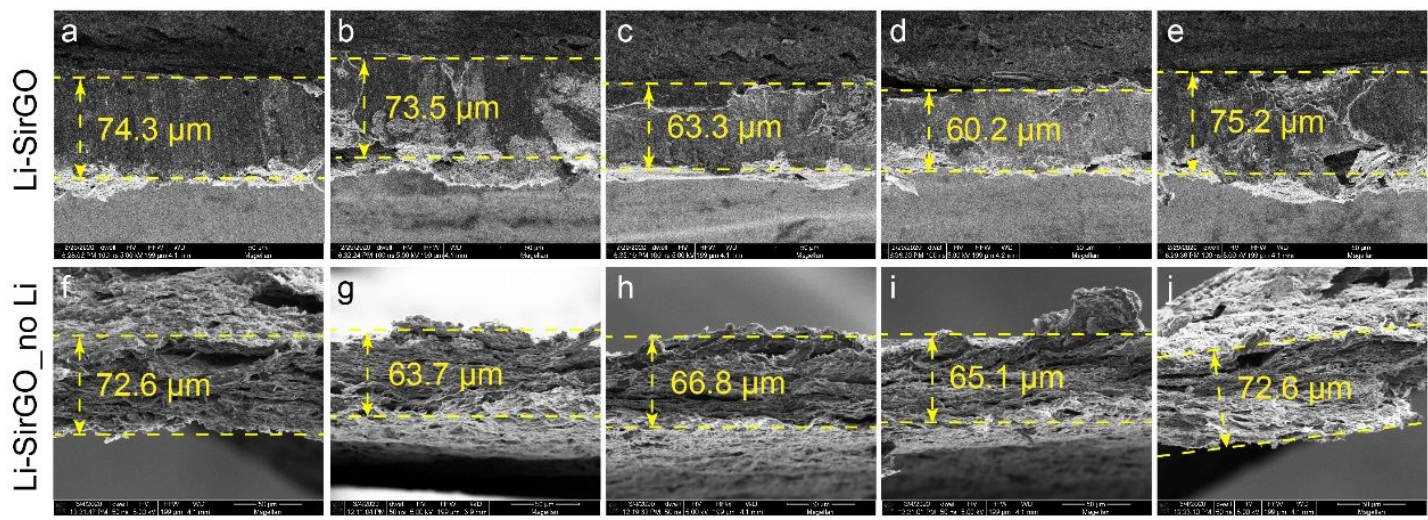

Figure S5. Thickness measurement of Li-SirGO films before and after metallic Li stripping. (a-e) Thickness of a Li-SirGO film at multiple regions measured through SEM. Cross-sections are produced by direct cutting of the film for clear edges. (f-j) Thickness of the same Li-SirGO film at multiple regions after metallic $\mathrm{Li}$ stripping till $0.4 \mathrm{~V}$ vs. $\mathrm{Li} / \mathrm{Li}^{+}$. Cross-sections are produced by tearing the film for clear observations of the layered structure after Li stripping. 

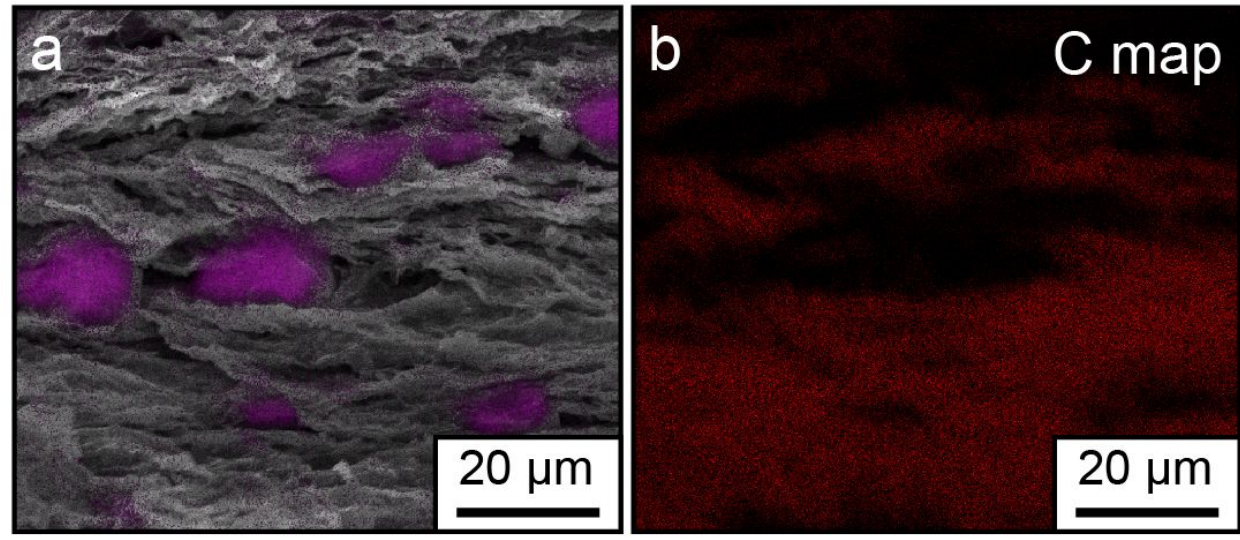

Figure S6. An overlay image of Figure 3e and $\mathrm{f}$ (a) and SEM-EDS mapping of $\mathrm{C}$ for the region shown in Figure 3e (b).
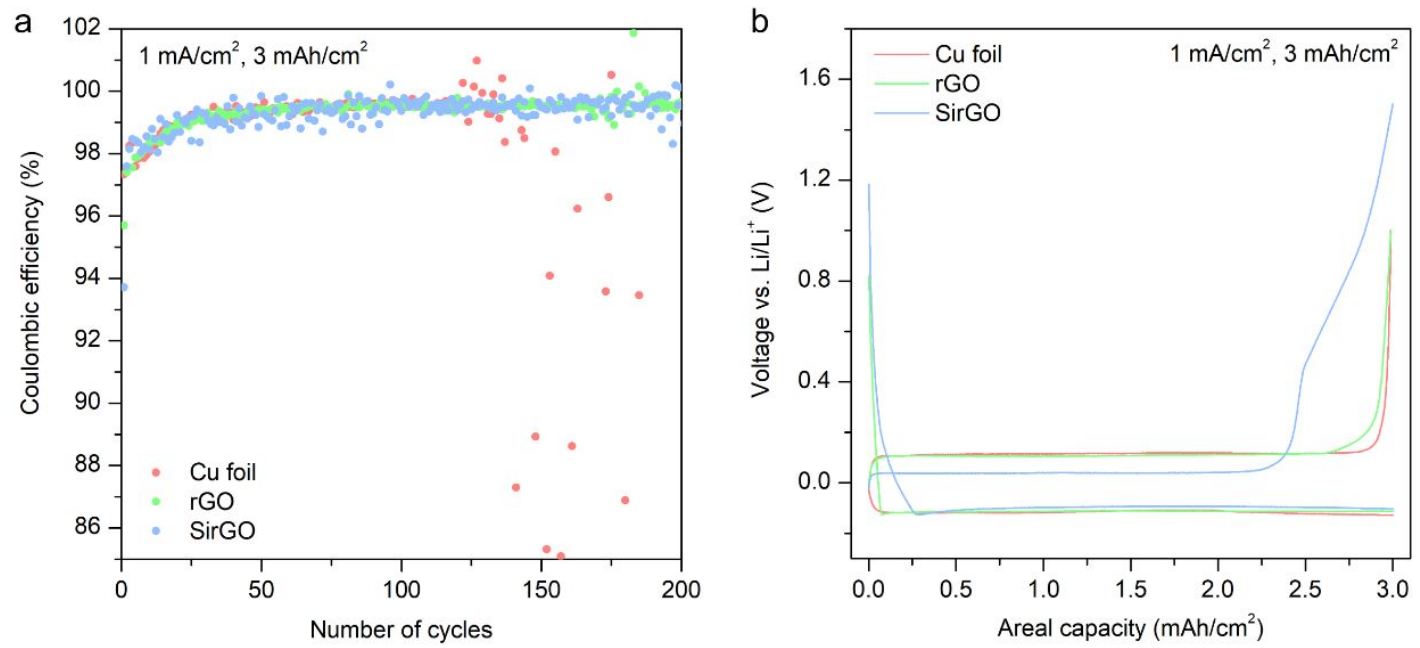

Figure S7. Coulombic efficiency comparison under $1 \mathrm{~mA} / \mathrm{cm}^{2}$ and $3 \mathrm{mAh} / \mathrm{cm}^{2} .1 \mathrm{M} \mathrm{LiFSI} / \mathrm{DME}-$ TFEO is used as the electrolyte.

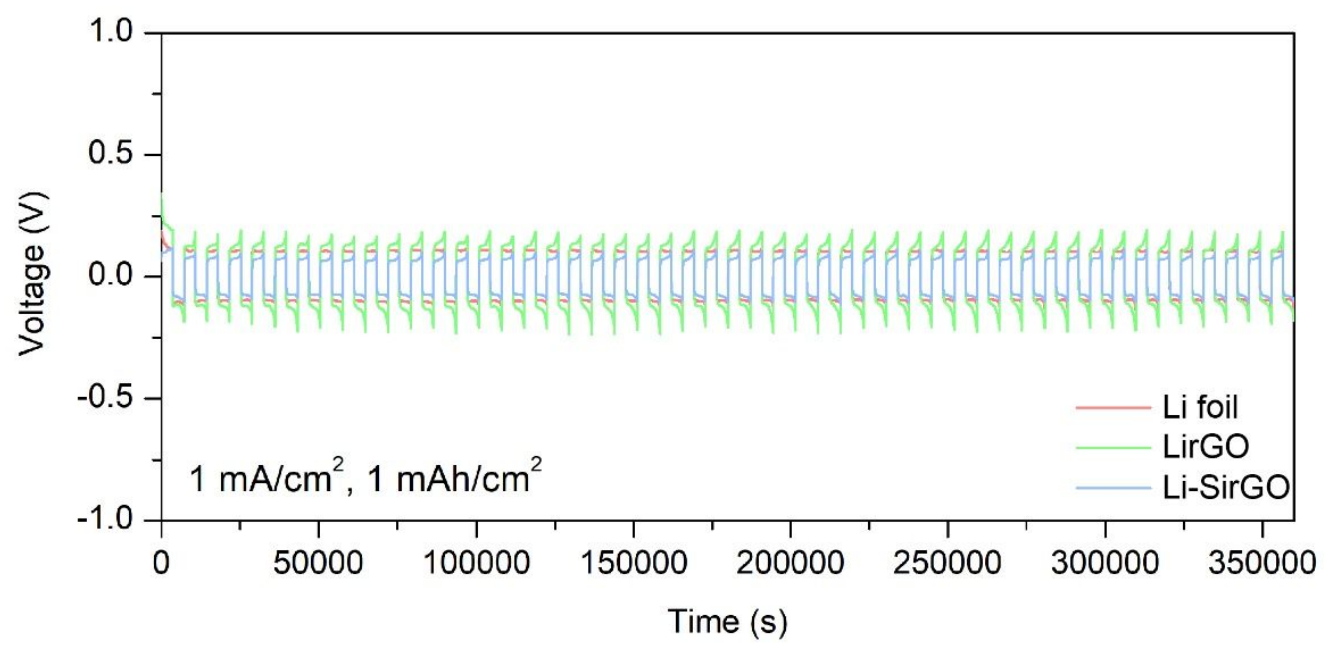

Figure S8. Symmetric cell cycling performance comparison in $1 \mathrm{M} \mathrm{LiFSI/DME-TFEO}$ electrolyte. 


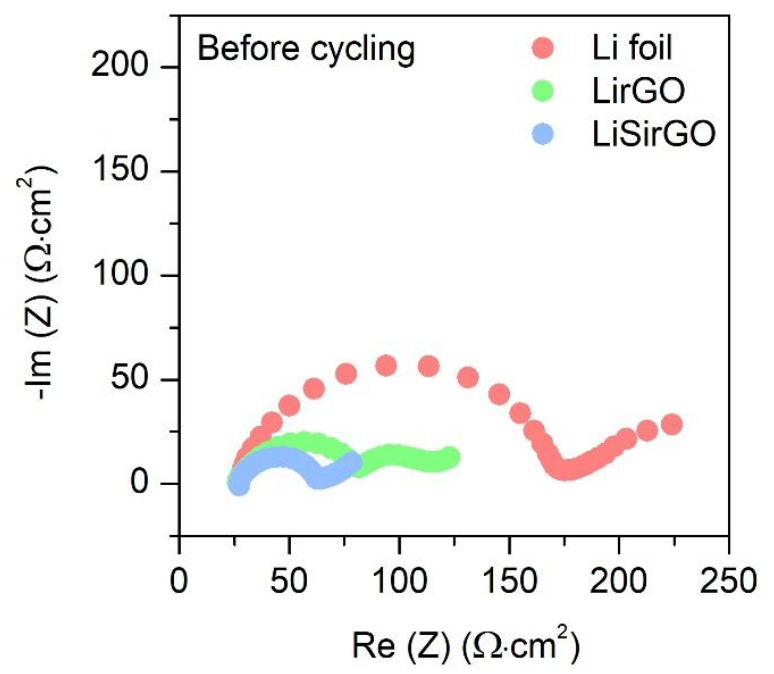

Figure S9. Impedance measurement of symmetric cells with $1 \mathrm{M}$ LiFSI/DME-TFEO electrolyte before cycling.
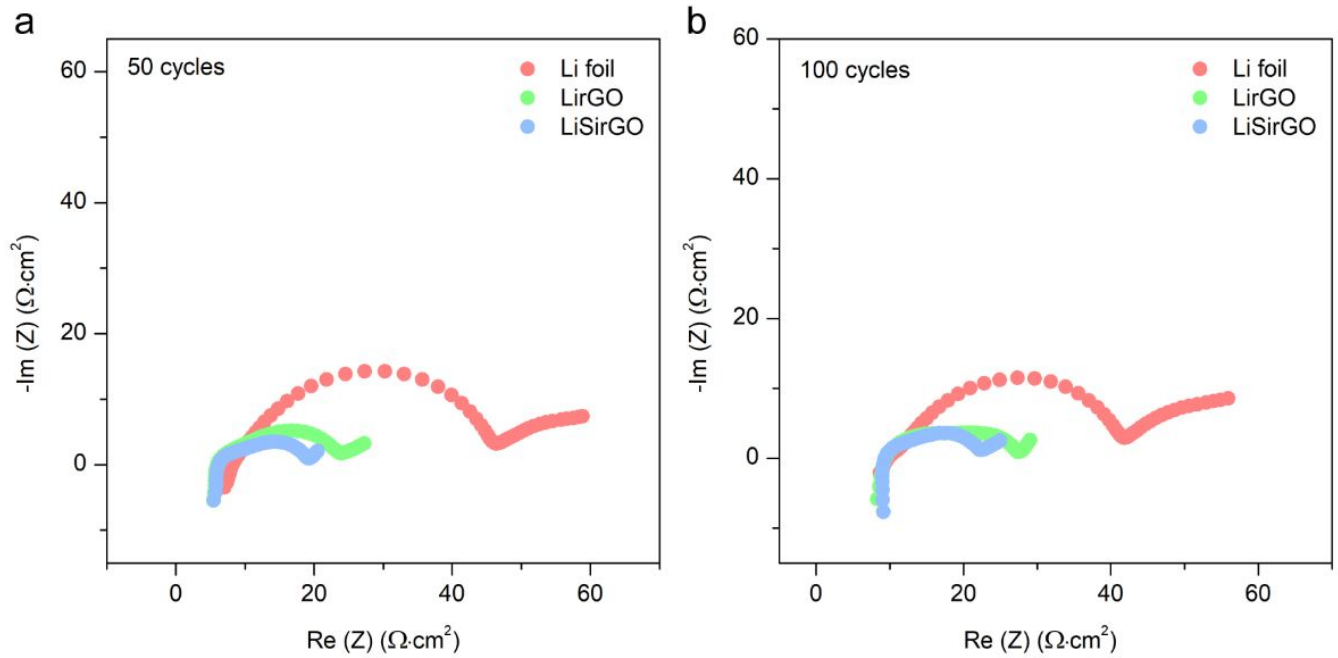

Figure S10. Impedance measurement of symmetric cells with $1 \mathrm{M} \mathrm{LiPF}_{6} / \mathrm{EC}-\mathrm{DEC}-\mathrm{FEC}-\mathrm{VC}$ electrolyte after 50 and 100 cycles. 

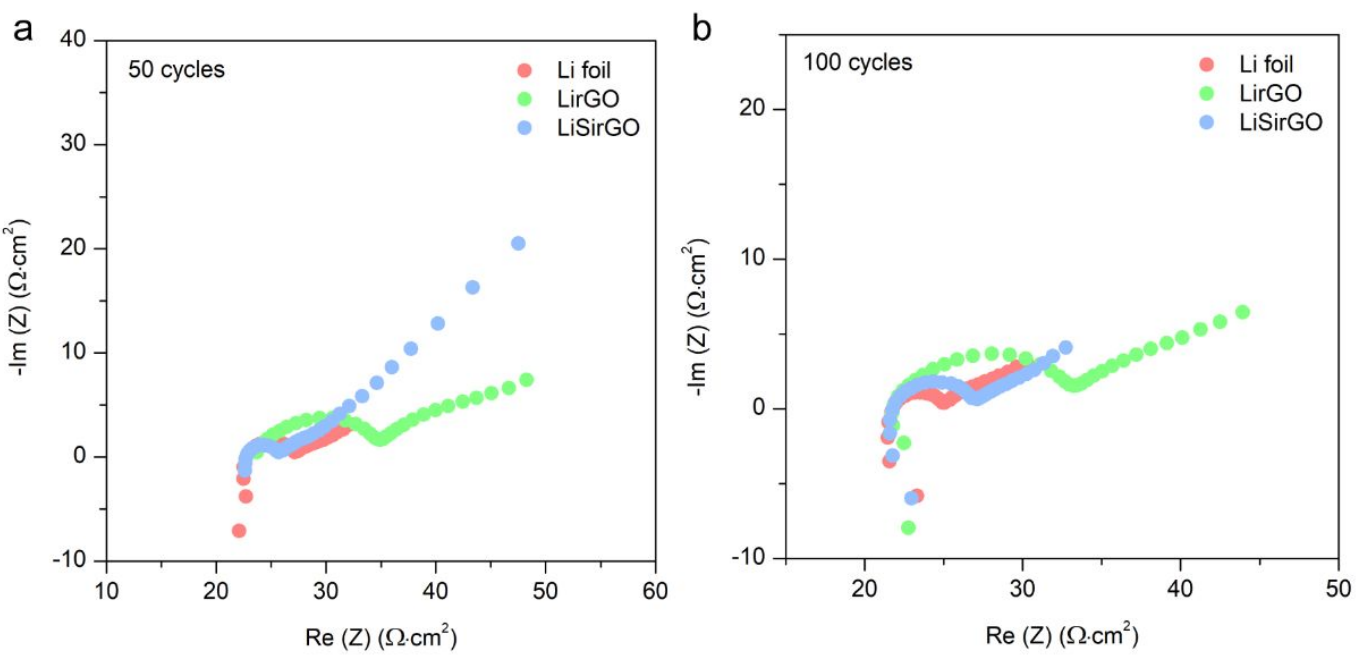

Figure S11. Impedance measurement of symmetric cells with $1 \mathrm{M}$ LiFSI/DME-TFEO electrolyte after 50 and 100 cycles.

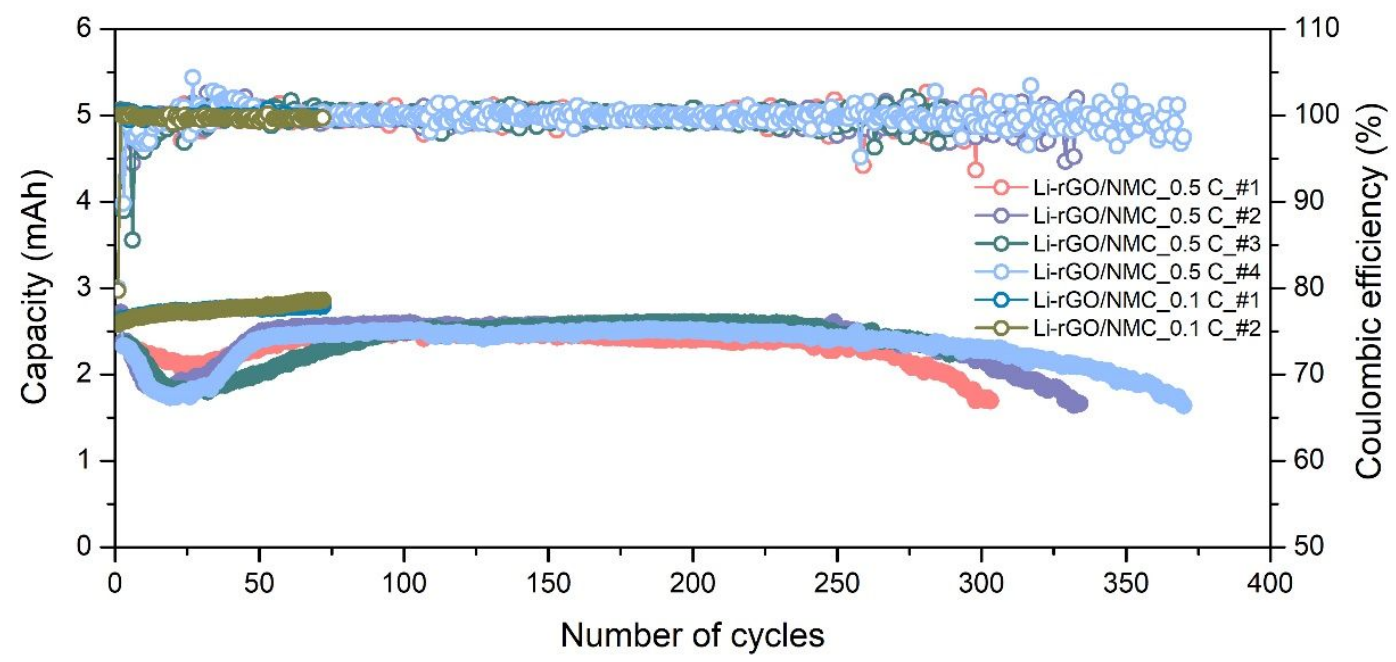

Figure S12. Repeatability test for the capacity drop and increase phenomenon in Li$\mathrm{rGO} \| \mathrm{NMC} 532$ full cells at the beginning of cycling. The $1 \mathrm{M}$ LiFSI/DME-TFEO electrolyte is used. 